\title{
Patient-to-Patient Hepatitis C Virus Transmissions Associated with Infection Control Breaches in a Hemodialysis Unit
}

Nicola D. Thompson ${ }^{1,2 *}$, Ryan T. Novak ${ }^{1,2}$, Mary Beth White-Comstock ${ }^{3}$, Guoliang Xia ${ }^{1}$, Lilia Ganova-Raeva ${ }^{1}$, Sumathi Ramachandran ${ }^{1}$, Yury Khudyakov $^{1}$, Stephanie R. Bialek ${ }^{1}$ and Ian T. Williams ${ }^{1}$

${ }^{1}$ Division of Viral Hepatitis, Centers for Disease Control and Prevention, Atlanta, GA, USA

${ }^{2}$ Epidemic Intelligence Service, Centers for Disease Control and Prevention, Atlanta, GA, USA

${ }^{3}$ Virginia Department of Health, Richmond, VA, USA

\begin{abstract}
Background: Three patients attending a hemodialysis unit were diagnosed with acute hepatitis C virus (HCV) infection. We investigated the scope and mode of transmission.
\end{abstract}

Methods: Patients and staff were tested to determine HCV infection status; all HCV-RNA-positive sera underwent quasispecies analysis to assess genetic relatedness. Staff practices were evaluated via interviews and observations. A cohort study was performed to assess risk factors for incident HCV infection.

Results: HCV infection was documented at time of hire or unit admission for 2 staff and 12 patients (prevalent case-patients). Seven (13\%) of 52 patients HCV susceptible at admission to the unit subsequently acquired HCV infection (incident case-patients). Analysis of HCV quasispecies from the hyper variable region 1 identified 2 separate clusters each containing 3 incident case-patients and 1 prevalent case-patient. Incident case-patients received a higher median number of intravenous medications per dialysis session compared to susceptible patients (2.1 vs 1.8 , $p$-value $=0.0606$ ). Only one incident case-patient received dialysis on the same machine as their genetically related prevalent case-patient. Preparation of injection medications at the dialysis station on a mobile medication cart, and failures to clean environmental surfaces between patients were infection control breaches identified as likely modes of HCV transmission.

Conclusions: Epidemiologic and laboratory data revealed transmission of HCV among patients at the same dialysis unit. Transmission was most likely related to infection control breaches. Our findings reinforce the risk of patient-to-patient HCV transmission in hemodialysis units when staff fails to adhere to recommended infection control practices.

Keywords: Hemodialysis; HCV; Infection control; Outbreak; Prevention

In the United States the prevalence of hepatitis C virus (HCV) infection among patients undergoing hemodialysis $(\sim 8-10 \%)$ is approximately 5 times higher than the general population $(1.6 \%)[1,2]$. Due to implementation of routine screening of the blood supply and virtual elimination of $\mathrm{HCV}$ transmission via blood transfusion [3] and the overall declining incidence of acute HCV infection in the United States [4], intra-facility $\mathrm{HCV}$ transmission has been increasingly recognized as cause of incident $\mathrm{HCV}$ infection in hemodialysis patients [5-9].

During a 3-month period in 2006, 3 patients attending the same hemodialysis unit became jaundiced and were subsequently diagnosed with acute HCV infection. All 3 were documented to be HCV antibody (anti-HCV) negative on admission to the unit and had remained anti-HCV negative during routine screening performed annually. In response, the facility initiated monthly anti-HCV testing to identify additional newly infected patients and contacted public health officials for assistance identifying potential causes of HCV infection. To determine the magnitude, source and mode of transmission and implement necessary prevention measures a public health investigation was initiated by the Virginia Department of Health and the Centers for Disease Control and Prevention (CDC).

\section{Materials and Methods}

\section{Case ascertainment}

Medical records were reviewed to identify the HCV status of all patients attending the hemodialysis unit, those anti-HCV negative at the time of admission to the unit and in January 2006 (during routine annual screening) were tested monthly for anti-HCV between May and July by the facility. In June 2006, sera was collected from all current hemodialysis patients and staff and sent to the Division of Viral Hepatitis Laboratory at the CDC for testing by enzyme immunoassay (EIA) (ORTHO HCV Version 3.0 EIA, Ortho-Clinical Diagnostics, Raritan, New Jersey) and HCV RNA testing by polymerase chain reaction (PCR) (AMPLICOR HCV Test, version 2.0, Roche Molecular Systems, Branchburg, New Jersey) with a lower limit of detection of $\sim 50$ copies $/ \mathrm{ml}$.

\section{Definitions}

The following case definitions were used to classify patients' $\mathrm{HCV}$ infection status. Patients with prevalent HCV infection (prevalent casepatients) were known to be anti-HCV positive upon first admission to the unit for treatment. Patients with incident HCV infection (incident

*Corresponding author: Nicola D. Thompson, 1600 Clifton Road, MS A-24 Atlanta, GA 30333, USA, Tel: 404-639-1668; Fax: 404-639-2647; E-mail ndthompson@cdc.gov

Received December 27, 2011; Accepted February 15, 2012; Published February 17,2012

Citation: Thompson ND, Novak RT, White-Comstock MB, Xia G, et al. (2012) Patient-to-Patient Hepatitis C Virus Transmissions Associated with Infection Control Breaches in a Hemodialysis Unit. J Nephrol Therapeutic S10:002. doi:10.4172/2161-0959.S10-002

Copyright: (c) 2012 Thompson ND, et al. This is an open-access article distributed under the terms of the Creative Commons Attribution License, which permits unrestricted use, distribution, and reproduction in any medium, provided the original author and source are credited. 
Citation: Thompson ND, Novak RT, White-Comstock MB, Xia G, et al. (2012) Patient-to-Patient Hepatitis C Virus Transmissions Associated with Infection Control Breaches in a Hemodialysis Unit. J Nephrol Therapeutic S10:002. doi:10.4172/2161-0959.S10-002

Page 2 of 5

case-patients) were known to be anti-HCV negative at the time admission to the unit but were subsequently found to be anti-HCV positive and/or HCV RNA positive. Patients were HCV susceptible if they were anti-HCV negative at the time of admission to the facility and remained anti-HCV negative and HCV RNA negative during all repeat tests performed through the end of the investigation. Patients were classified as having unknown HCV status if they were know to be anti-HCV negative at the time admission to the unit but were not tested as part of the investigation (e.g., deceased, transferred, or otherwise lost to follow-up).

\section{Quasispecies analysis}

The HCV genotype for all HCV RNA positive specimens was determined from the sequence of a 300-nucleotide NS5B coding region. Genetic relatedness of the virus was assessed by analysis of $\mathrm{HCV}$ quasispecies (the population of related HCV variants that occur within infected individuals). The quasispecies were analyzed by sequencing a segment amplified from the E1-hypervariable region 1 (HVR1) of the HCV genome using methods previously described [10]. The HCV quasispecies obtained from patients and staff at the hemodialysis unit were compared to each other, and also to the quasispecies of selected sequences, with the same HCV genotype and $>95 \%$ nucleotide identity in the NS5B coding region, from HCV-infected individuals from the Third National Health and Nutrition Examination Survey (NHANES III) - a representative sample of the non-institutionalized civilian population of the United States [11].

\section{Epidemiologic Investigation and Cohort Study}

Hemodialysis treatment records, patient medical charts, and laboratory records including anti-HCV, hepatitis B virus (HBV) test results, and monthly liver enzyme test results for all patients who attended the dialysis unit were reviewed. Incident case-patients were interviewed by a public health official using a standardized questionnaire to elicit the presence of potential risk factors for HCV infection (e.g., history of illegal and injection drug use, sexual history, sexual or household contact with a person with HCV infection, and history of medical care received outside of the hemodialysis unit). To identify potential risk factors for incident $\mathrm{HCV}$ infection we performed a retrospective cohort study, including only incident case-patients and HCV susceptible patients dialyzed between November 1, 2005 and May $31^{\text {st }} 2006$ (the period during which incident case-patients were most likely exposed to HCV) [12]. Data collected included patient demographics, dialysis treatment characteristics, dialysis schedule, shift, station, and machine.

\section{Statistical Methods}

Patient demographic and dialysis-specific characteristics were compared using Fishers exact test for categorical variables and the nonparametric Kruskall-Wallis test for comparing the median of continuous variables. Potential dialysis-related risk factors, were compared using attack rates, defined as the number of incident HCV cases divided by the total number of patients with and without the risk factor assessed, and risk ratios (RR) and 95\% confidence intervals (CI). All tests were 2 -sided and $p$-values of $<.05$ were considered statistically significant. Statistical analysis was performed using SAS, version 9.2.

\section{Environmental and infection control assessment}

Inspections of the hemodialysis unit and observation of hemodialysis treatment and patient care practices was performed on multiple days and dialysis shifts. Infection control training manuals and facility policies, machine maintenance and cleaning schedules and dialyzer reprocessing logs were reviewed. Staff was interviewed about their routine dialysis and infection control practices at the facility.

\section{Results}

The dialysis unit was an outpatient community-based facility, with approximately 66 patients regularly attending each week, and treatment provided by 16 patient care staff. The unit had 17 dialysis stations, with one dedicated for the isolation of patients with HBV infection. All patients were routinely tested for anti-HCV upon admission to the unit and then annually, usually in January. All patients tested for antiHCV in January 2006 were negative. HBV serologic testing at the unit followed CDC recommendations [13], and patients serum aspartate aminotransferase (AST) levels determined monthly. Monitoring of patient alanine aminotransferase (ALT) levels was not performed at this hemodialysis unit. Patients generally attend the unit 3 times each week on a Monday, Wednesday and Friday (MWF) schedule, or Tuesday, Thursday and Saturday (TTS) schedule, during one of three daily shifts, morning (shift 1), mid-day (shift 2) or afternoon (shift 3). The median age of patients was 67 years (inter quartile range [IQR] 52 - 75 years), $42 \%$ were male and $55 \%$ had diabetes mellitus. The median length of time receiving hemodialysis was of 38 months (IQR $16-75$ months).

\section{HCV infection status of patients and staff}

Based on past test results documented in patient charts and testing performed during the investigation, 2 patients had unknown HCV status (transferred to another unit prior to investigation), 12 patients were classified as having prevalent $\mathrm{HCV}$ infection (18\%), 45 were HCV susceptible, and 7 had incident HCV infection. One patient had chronic HBV infection, and no new HBV infections were identified via review of routine testing. Among the 16 patient care staff tested for HCV in June 2006, 14 were anti-HCV and HCV RNA negative and 2 had HCV infection. Both had past diagnoses of chronic HCV infection prior to employment at the facility.

Three (43\%) of 7 patients with incident HCV infection had symptoms consistent with acute $\mathrm{HCV}$ infection (i.e., jaundice and malaise); 2 required hospitalization one of whom died from an unrelated cause. Three patients were found to have incident HCV infection only during monthly HCV screening implemented by the facility, and the seventh patient was consistently anti-HCV negative but was found to be HCV RNA positive in June 2006 (Figure 1). Six of 7 incident case-patients had a serum AST levels increase greater than the upper limit of normal (>38 unit/L) between January - May 2006 (median peak AST: 99 units/L; range: 51 - 758 units/L), but only 3 had an increase $>100$ units/L. Via patient interview and chart review, no behavioral risk factors for HCV infection or other common exposure opportunities outside of the hemodialysis unit were identified for incident case-patients.

\section{Laboratory investigation and quasispecies analysis}

Six of 7 patients with incident HCV infection were tested for HCVRNA and found to be positive with HCV genotype $1 a$; the seventh died and no serum was available for HCV RNA testing. Ten of 12 patients with prevalent HCV infection were HCV-RNA-positive; 7 were HCV genotype $1 a, 2$ were genotype $1 b$, and 1 genotype $2 b$. Both staff with chronic HCV infection were HCV-RNA-positive and HCV genotype $1 a$.

E1-HVR1 quasispecies analysis was performed on the $15 \mathrm{HCV}$ genotype 1a specimens ( 6 incident, 7 prevalent, 2 staff), and two highly related clusters were identified. For cluster A (3 incident and 
Citation: Thompson ND, Novak RT, White-Comstock MB, Xia G, et al. (2012) Patient-to-Patient Hepatitis C Virus Transmissions Associated with Infection Control Breaches in a Hemodialysis Unit. J Nephrol Therapeutic S10:002. doi:10.4172/2161-0959.S10-002

Page 3 of 5

1 prevalent) the maximum HCV quasispecies sequence identity was $99.3 \%$ to $100 \%$, and for cluster B ( 3 incident, 1 prevalent) the maximum HCV quasispecies sequence identity was $97.3 \%$ to $100 \%$ (Figure 2). No genetic relatedness was identified between these two clusters and any of the 5 NHANES III participants with genotype 1 a HCV infection (the maximum sequence relatedness $84.9 \%-91.8 \%$ and $84.2 \%-93.1 \%$, respectively for cluster $\mathrm{A}$ and $\mathrm{B})$. For the two staff with genotype $1 \mathrm{a}$ $\mathrm{HCV}$ infection, the maximum relatedness was $89.7 \%-90.4 \%$ and 90.7\%-91.1\% for cluster $\mathrm{A}$, and $82.8-83.2 \%$ and $84.5 \%-85.2 \%$ for cluster $\mathrm{B}$, indicating virus from $\mathrm{HCV}$ infected staff was not related to the genotype $1 a \mathrm{HCV}$ infection among patients.

\section{Cohort study}

The retrospective cohort study included 7 incident case-patients and $45 \mathrm{HCV}$ susceptible patients. Attack rates did not differ significantly by age, gender, race, primary cause of renal failure, presence of diabetes, or length or of time receiving dialysis (Table 1). The HCV attack rates were higher for patients dialyzed on the MWF schedule and on shifts 2

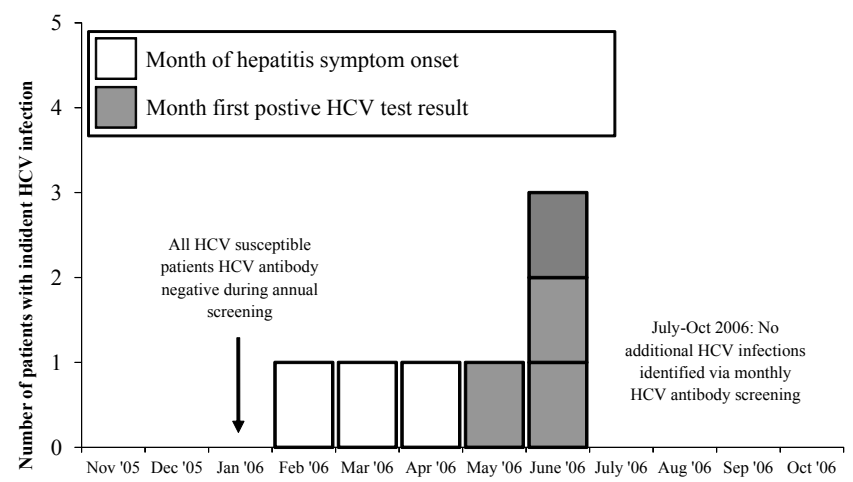

Month and Year

Figure 1: Hemodialysis patients with incident hepatitis $\mathrm{C}$ virus (HCV) infection by month of diagnosis and method of detection.



Figure 2: Phylogenetic tree of hepatitis $\mathrm{C}$ virus (HCV) E1 hypervariable region sequences (quasispecies) from six patients with incident genotype 1a HCV infection, and seven patients and two staff with prevalent genotype 1a HCV infection from the dialysis unit, compared to five Third National Health and Nutrition Examination participants with genotype 1a HCV infection. Maximum HCV quasispecies sequence identity for cluster A $99.3 \%$ to $100 \%$, and $97.7 \%$ to $100 \%$ for cluster $\mathrm{B}$. and 3 (compared to shift 1), and were also higher for patients that that more frequently (greater than the median value for the cohort) received intravenous (IV) epogen alfa, iron sucrose, paricalcitol and sodium chloride during their dialysis sessions, however, these differences were not statistically significant (Table 1). When the number of IV drugs received per treatment session were combined, incident case-patients received more IV drugs per dialysis session compared to susceptible patients (2.1 vs 1.8, respectively; Wilcoxon rank sum, $\mathrm{p}=0.0606$ ).

\section{Evaluation of shared dialysis machines}

To evaluate the potential for HCV transmission via the dialysis machine, shared use of machines was assessed for the 6 incident casepatients and 2 prevalent case-patients identified in each genetically related cluster. In cluster A, all 3 incident case-patients were dialyzed on the shift following directly after the prevalent case-patient. Two prevalent case-patients were never dialyzed on the same machine after the prevalent case-patient, and the third dialyzed on same machine during only 4 dialysis sessions. In cluster B, all 3 incident case-patients were dialyzed on the same shift as the prevalent case-patient and at adjacent stations and were never dialyzed on the same machine.

\section{Environmental and Infection Control Assessment}

Interviews and observations of practices and the patient-care environment were performed approximately 6 months after the first patient was diagnosed with acute HCV infection. Staff hand hygiene, glove, gown and face-shield use was appropriate. No opportunities for $\mathrm{HCV}$ transmission were identified with respect to dialyzer reprocessing. Disposable, single-use cloths soaked in a $1 \%$ bleach solution were used to wipe down the dialysis station and machine between patients. Although, technicians were observed wiping down machine and station surfaces while the current patient remained in the station chair awaiting homeostasis. The contact time for bleach to disinfect surfaces was limited to a few seconds.

During our observations, injectable medications were kept at medication stations located at each end of the unit. However, they were located directly next to and within splashing distance of the biohazard bins for discarding used tubing, and plastic containers storing clean supplies at the medication station were observed to have dried blood splashes. No facility protocol was in place regarding routine cleaning and disinfection for medication stations. Medication vials labeled as single-use, specifically epoetin alpha, appeared to have been used as multi-dose vials, as multiple puncture holes were observed in open single-dose vials at the medication station. Interviews with staff revealed that up until the month prior to the investigation, the facility had routinely used a mobile medication cart to store and prepare medications. The cart was transported between patients to each dialysis station, where injection medications were prepare and administer to patients.

\section{Discussion}

We identified patient-to-patient HCV transmission within a hemodialysis unit, most likely attributable to failures to follow recommended infection control practices. No HCV risk factors outside the unit were indentified for incident case-patients, and incident casepatients were found by quasispecies analysis to have the same virus as two prevalent case-patients also attending the unit. Use of single dose medication vials for multiple patients along with vial preparation at the patient station, storage of clean supplies near biohazard disposal areas, and failures to adequately clean and disinfect dialysis machines and station between each patient are all plausible mechanisms of HCV 
Citation: Thompson ND, Novak RT, White-Comstock MB, Xia G, et al. (2012) Patient-to-Patient Hepatitis C Virus Transmissions Associated with Infection Control Breaches in a Hemodialysis Unit. J Nephrol Therapeutic S10:002. doi:10.4172/2161-0959.S10-002

Page 4 of 5

\begin{tabular}{|c|c|c|c|c|c|c|c|}
\hline \multirow{2}{*}{ Risk factor } & \multicolumn{3}{|l|}{ Exposed } & \multicolumn{3}{|l|}{ Unexposed } & \multirow{2}{*}{ Risk Ratio $(95 \% \mathrm{Cl}) ;$ p-value ${ }^{1}$} \\
\hline & Incident Cases & Total patients & Attack rate $\%$ & Incident Cases & Total patients & Attack rate $\%$ & \\
\hline Age $>68$ years $^{2}$ & 3 & 24 & 12.5 & 4 & 28 & 14.3 & $0.875(0.217-3.527) ;>0.999$ \\
\hline Male gender & 4 & 17 & 23.5 & 3 & 35 & 8.6 & $2.745(0.691-10.910) ; 0.295$ \\
\hline Race: White & 4 & 15 & 26.7 & 3 & 38 & 7.9 & $3.378(0.856-13.320) ; 0.179$ \\
\hline Cause of renal failure: Hypertension & 4 & 35 & 11.4 & 3 & 17 & 17.6 & $0.648(0.163-2.575) ; 0.827$ \\
\hline Diabetes mellitus & 2 & 28 & 7.1 & 5 & 22 & 22.7 & $0.314(0.067-1.469) ; 0.245$ \\
\hline Dialysis $>34$ months $^{2}$ & 3 & 25 & 12 & 4 & 27 & 14.8 & $0.810(0.201-0.267) ;>0.999$ \\
\hline Dialyzed on MWF schedule & 6 & 30 & 20.0 & 1 & 22 & 4.3 & 4.400 (0.570 - 33.980); 0.226 \\
\hline Dialyzed on $1^{\text {st }}$ shift & 1 & 25 & 4.0 & 6 & 27 & 22.2 & $0.180(0.023-1.392) ; 0.124$ \\
\hline Received epogen alpha $^{3}$ & 4 & 26 & 15.4 & 3 & 26 & 11.5 & $1.333(0.331-5.378) ;>0.999$ \\
\hline Received paricalcitol $^{3}$ & 5 & 27 & 18.5 & 2 & 25 & 8.0 & $2.315(0.493-10.870) ; 0.486$ \\
\hline Received iron sucrose $^{3}$ & 4 & 26 & 15.4 & 3 & 26 & 11.5 & $1.333(0.331-5.378) ;>0.999$ \\
\hline Received sodium chloride $^{3}$ & 4 & 26 & 15.4 & 3 & 26 & 11.5 & $1.333(0.331-5.378) ;>0.999$ \\
\hline
\end{tabular}

$\mathrm{Cl}$ : Confidence Interval

MWF: Monday, Wednesday, and Friday

Attack Rate: Number incident cases / number total patients $x 100$

1: Two-sided Fisher exact $p$-value

2: Cohort median

3: Received greater than cohort median value

Table 1: Risk for hepatitis $\mathrm{C}$ virus infection among cohort of 7 incidence case-patients and $45 \mathrm{HCV}$ susceptible patients according to selected patient and hemodialysis treatment risk factors, November 2005 to May 2006

transmission that we identified. Each of these practices can facilitate cross-contamination and have previously been implicated in patientto-patient HCV transmission in hemodialysis and other healthcare settings $[6,7,9,14-16]$.

Due to concurrent treatment of hemodialysis patients in a shared area, the risk for blood contamination of the physical environment, equipment (e.g., healthcare hands, gloves, needles and syringes), and medication vials is high [12]. To reduce the risk of bacterial and viral contamination of medication vials, CDC recommends vials and injection equipment are stored and prepared in a clean environment away from the immediate patient treatment area, that medications packaged as single-use be dedicated to single patient use, and that medications packaged as multidose be assigned to single patients whenever possible [12,14,17-19,20]. Maintaining these basic infection control practices is essential for providers to prevent blood borne pathogen transmission when storing, preparing and administering medications from vials [16-18].

Through the combined laboratory and epidemiologic approach to this investigation we were able to thoroughly evaluate the potential for HCV transmission via the dialysis machines, a mechanism previously proposed [21], yet rarely conclusively demonstrated [6]. Our analysis identified that only one incident case-patient was dialyzed on the same machine directly after their genetically related prevalent case-patient (i.e., their HCV source). Even though, the shared use of machines could not be assessed for one patient (from whom HCV RNA was not obtained), we ruled out HCV transmission via the dialysis machine as mechanism of HCV transmission in this investigation. Interestingly, being dialyzed on the same shift or on a following shift and at adjacent stations was common. This suggests indirect contact transmission, either via shared equipment or supplies or via the hands of healthcare workers, likely contributed to $\mathrm{HCV}$ transmission between patients within each cluster.

At this unit patients' AST levels were measured monthly, however, liver enzyme elevations that preceded diagnosis of incident HCV infection were generally modest and overlooked. Because monitoring of ALT is more specific for the detection of HCV infection than AST, the CDC recommends monthly monitoring of ALT levels [12,13]. This should be performed in conjunction with semi-annual HCV screening
[13], as monitoring of liver enzymes alone has been shown to be an inadequate method of detecting new HCV infection in hemodialysis populations [22]. Because most people newly infected with HCV are asymptomatic [22], performing baseline (upon unit admission) and routine semi-annual HCV-screening in hemodialysis patients is necessary to identify new infections that may otherwise go unrecognized $[6,12]$. The detection of a new, confirmed positive anti-HCV test result should prompt referral of the patient to a medical provider for evaluation and appropriate management of the infection. As well as an evaluation of the potential for intra-facility HCV transmission by assessing facility infection control practices and patient risk factors in collaboration with public health officials $[12,23]$. As recently reported, failures to implement routine HCV screening programs can result in prolonged periods of undetected HCV transmission [7].

The main limitation of this investigation is that we were unable to clearly identify the mode of HCV transmission, one common to HCV investigations in hemodialysis settings. This is likely because our investigation began many months after first patient was diagnosed with acute HCV infection, the likely exposure period was long (7 months), and because hemodialysis patients encountered multiple potential exposure opportunities. We did, however, identify several breaches in infection control practice that are known to facilitate HCV transmission in healthcare settings, and upon correction of these practices, no additional $\mathrm{HCV}$ transmissions have been identified at this unit.

Our findings suggest that apparent failures to follow existing infection control recommendations resulted in patient-to-patient HCV transmission, most likely through contamination of shared medication vials or of environmental surfaces and not via shared use of the same dialysis machine. Additionally, this investigation highlights the value of routine HCV screening programs to identify new HCV infections and alert staff to potential HCV transmission among patients. Preventing healthcare-associated infections, in hemodialysis patients is a public health priority, as demonstrated by the recent publication of the US Department of Health and Human Services action plan to prevent healthcare-associated infections in End Stage Renal Disease Facilities [24], which includes a focus on HCV infection prevention. Adherence to existing infection control precautions and routine HCV screening 
Citation: Thompson ND, Novak RT, White-Comstock MB, Xia G, et al. (2012) Patient-to-Patient Hepatitis C Virus Transmissions Associated with Infection Control Breaches in a Hemodialysis Unit. J Nephrol Therapeutic S10:002. doi:10.4172/2161-0959.S10-002

Page 5 of 5

remain the cornerstone of preventing $\mathrm{HCV}$ transmission among hemodialysis patients.

\section{Acknowledgements \& Disclosures}

We would like to thank the staff and patients of the hemodialysis facility for their cooperation during the field investigation, and Mr. Timothy Powell and Dr. Diane Woolard of the Virginia Department of Health for their support during the field investigation.

The findings and conclusions in this report are those of the authors and do not necessarily represent the official position of the Centers for Disease Contro and Prevention.

\section{References}

1. Fissell RB, Bragg-Gresham JL, Woods JD, Jadoul M, Gillespie B, et al. (2004) Patterns of hepatitis $\mathrm{C}$ prevalence and seroconversion in hemodialysis units from three continents: The DOPPS. Kidney Int 65: 2335-2342.

2. Finelli L, Miller JT, Tokars JI, Alter MJ, Ardunio MJ (2005) National surveillance of dialysis-associated diseases in the United States, 2002. Semin Dial 18: 5261.

3. Busch MP, Kleinman SH, Nemo GJ (2003) Current and Emerging Infectious Risks of Blood Transfusions. JAMA 289: 959-962.

4. Wasley A, Grytdal S, Gallagher K (2008) Surveillance for acute viral hepatitisUnited States, 2006. MMWR Surveill Summ 57: 1-24

5. Savey A, Simon F, Izopet J, Lepoutre A, Fabry J, et al. (2005) A Large nosocomial outbreak of hepatitis $\mathrm{C}$ virus infections at a hemodialysis center. Infect Control Hosp Epidemiol 26:752-760.

6. Kidney Disease: Improving Global Outcomes (2008) Guideline 3: Preventing HCV transmission in hemodialysis units. Kidney Int. 2008 73: 46-52.

7. Centers for Disease Control and Prevention (CDC) (2009) Hepatitis C Virus transmission at an outpatient hemodialysis unit - New York, 2001-2008. MMWR Morb Mortal Wkly Rep 58:189-94.

8. Thompson ND, Novak RT, Datta S, Cotter S, Arduino MJ, et al. (2009) Hepatitis $C$ virus transmission in the hemodialysis units: importance of infection contro practices and aseptic technique. Infect Control Hosp Epidemiol 30: 900-933.

9. Rao A, Luckman E, Wise M (2010) Outbreak of Hepatitis C Virus Infections at an Outpatient Hemodialysis Facility [Abstract]. Presented at 5th Decennial International Conference on Healthcare-Associated Infections. Atlanta, GA USA March 20, 2010.

10. Ramachandran S, Xia G, Ganova-Raeva LM, Nainan OV, Khudyakov Y (2008) End-point limiting-dilution real-time PCR assay for evaluation of hepatitis $C$ virus quasispecies in serum: Performance under optimal and suboptimal conditions. J Virol Meth 151: 217-224.

11. Alter MJ, Kruszon-Moran D, Nainan OV, McQuillan GM, Gao F, et al. (1999) The prevalence of hepatitis $C$ virus infection in the United States, 1988 through 1994. N Engl J Med 341:556-562.

12. Jay H Hoofnagle (2002) Course and Outcome of Hepatitis C. Hepatology 36 21-29.

13. Centers for Disease Control and Prevention (CDC) (2001) Recommendations for preventing transmission of infections among chronic hemodialysis patients MMWR Recomm Rep 50: 1-43.

14. Kamili S, Krawczynski K, McCaustland K, Li X, Alter MJ (2007) Infectivity of hepatitis $C$ virus in plasma after drying and storing at room temperature. Infect Control Hosp Epidemiol 28: 519-524.

15. Caramelo C, de Sequera P, Lopez MD, Ortiz A (1999) Hand-born mechanism of dissemination of hepatitis $C$ virus in dialysis units: basis for new addenda to the present prevention strategies. Clin Nephrol 51: 59-60.

16. Girou E, Chevaliez S, Challine D, Thiessart M, Morice Y, et al. (2008) Determinant roles of environmental contamination and noncompliance with Standard Precautions in the risk of hepatitis $C$ virus transmission in a hemodialysis unit. Clin Infect Dis 47:627-633.

17. Patel PR, Larson AK, Castel AD, Ganova-Raeva LM, Myers RA, et al. (2006) Hepatitis $C$ virus infections from a contaminated radiopharmaceutical used in myocardial perfusion studies. JAMA 296: 2005-2011.

18. Centers for Disease Control and Prevention (CDC) Acute hepatitis $C$ virus infections attributed to unsafe injection practices at an endoscopy clinicNevada, 2007. MMWR Morb Mortal Wkly Rep 57: 513-517.

19. Siegel J, Rhinehart E, Jackson M, Chiarello L (2007) 2007 Guidelines fo Isolation Precautions: Preventing Transmission of Infectious Agents in Healthcare Settings.

20. Grohskopf LA, Roth VR, Feikin DR, Arduino MJ, Carson LA, et al. (2001) Serratia liquefaciens bloodstream infections from contamination of epoetin alfa at a hemodialysis center. N Engl J Med 344:1491-1497.

21. CDC. Guidelines For Viral Hepatitis Surveillance And Case Management [ACcessed July 23, 2011]

22. Saab S, Martin P, Brezina M, Gitnick G, Yee HF Jr (2001) Serum alanine aminotransferase in hepatitis $\mathrm{C}$ screening of patients on hemodialysis. Am J Kidney Disease 37: 308-315

23. Fabris P, Fleming VM, Giordani MT, Barnes E (2008) Acute hepatitis C: Clinical aspects, diagnosis, and outcome of acute HCV infection. Curr Pharm Des 14 1661-1665.

24. US Department of Health and Human Services (HHS). HHS Action Plan to Prevent Healthcare-Associated Infections: End-Stage Renal Disease Facilities. [Accessed Dec 12, 2011].

25. Delarocque-Astagneau E, Baffoy N, Thiers V, Simon N, de Valk H, et al. (2002) Outbreak of hepatitis $C$ virus infection in a hemodialysis unit: potentia transmission by the hemodialysis machine?. Infect Control Hosp Epidemiol 23 328-334.

26. Izopet J, Pasquier C, Sandres K, Puel J, Rostaing L (1999) Molecular evidence of noscomial transmission of hepatitis $\mathrm{C}$ virus in a French hemodialysis unit. $J$ Med Virol 58:139-144.

27. Katsoulidou A, Paraskevis D, Kalapothaki V, Arvanitis D, Karayiannis P et al. (1999) Molecular epidemiology of a hepatitis C virus outbreak in a haemodialysis unit. Multicenter Haemodialysis Cohort Study on Viral Hepatitis. Nephrol Dial Transplant. 14: 1188-1194.

28. Centers for Disease Control and Prevention (CDC) (2008) Infection contro requirements for dialysis facilities and clarification regarding guidance on parenteral medication vials. MMWR Morb Mortal Wkly Rep 57: 875-876. 